IJMMS 29:7 (2002) 375-380

PII. S0161171202007767

http://ijmms.hindawi.com

(c) Hindawi Publishing Corp.

\title{
THE GALOIS EXTENSIONS INDUCED BY IDEMPOTENTS IN A GALOIS ALGEBRA
}

\author{
GEORGE SZETO and LIANYONG XUE
}

Received 7 June 2001

\begin{abstract}
Let $B$ be a Galois algebra with Galois group $G, J_{g}=\{b \in B \mid b x=g(x) b$ for all $x \in B\}$ for each $g \in G, e_{g}$ the central idempotent such that $B J_{g}=B e_{g}$, and $e_{K}=\sum g \in K, e_{g} \neq 1 e_{g}$ for a subgroup $K$ of $G$. Then $B e_{K}$ is a Galois extension with the Galois group $G\left(e_{K}\right) \quad(=\{g \in G \mid$ $\left.g\left(e_{K}\right)=e_{K}\right\}$ ) containing $K$ and the normalizer $N(K)$ of $K$ in $G$. An equivalence condition is also given for $G\left(e_{K}\right)=N(K)$, and $B e_{G}$ is shown to be a direct sum of all $B e_{i}$ generated by a minimal idempotent $e_{i}$. Moreover, a characterization for a Galois extension $B$ is shown in terms of the Galois extension $B e_{G}$ and $B\left(1-e_{G}\right)$.
\end{abstract}

2000 Mathematics Subject Classification: 16S35, 16W20.

1. Introduction. The Boolean algebra of idempotents for commutative Galois algebras plays an important role (see [1, 3, 6]). Let $B$ be a Galois algebra with Galois group $G$ and $J_{g}=\{b \in B \mid b x=g(x) b$ for all $x \in B\}$ for each $g \in G$. Then, in [2], it was shown that the ideal $B J_{g}=B e_{g}$ for some central idempotent $e_{g}$. By using the Boolean algebra of central idempotents $\left\{e_{g}\right\}$ in the Galois algebra $B$, the following structure theorem of $B$ was shown. There exist some subgroups $H_{i}$ of $G$ and minimal idempotents of $\left\{e_{i} \mid i=\right.$ $1,2, \ldots, m$ for some integer $m\}$ such that $B=\oplus \sum_{i=1}^{m} B e_{i} \oplus B\left(1-\sum_{i=1}^{m} e_{i}\right)$ where $B e_{i}$ is a central Galois algebra with Galois group $H_{i}$ for each $i=1,2, \ldots, m$, and $B\left(1-\sum_{i=1}^{m} e_{i}\right)$ is $C\left(1-\sum_{i=1}^{m} e_{i}\right)$, a commutative Galois algebra with Galois group induced by and isomorphic with $G$ in case $1 \neq \sum_{i=1}^{m} e_{i}$ where $C$ is the center of $B$. Let $\left(B_{a} ; \dot{+}, \cdot\right)$ be the Boolean algebra generated by $\left\{0, e_{g} \mid g \in G\right\}$ where $e \cdot e^{\prime}=e e^{\prime}$ and $e \dot{+} e^{\prime}=e+e^{\prime}-e e^{\prime}$ for any $e$ and $e^{\prime}$ in $B_{a}$. In the present paper, we study the Galois extension $B e_{K}$ where $e_{K}=\sum_{g \in K, e_{g} \neq 1} e_{g} \in B_{a}$ for a subgroup $K$ of $G$. Let $G(e)=\{g \in G \mid g(e)=e\}$ for a central idempotent $e$. Then it will be shown that $K \subset N(K) \subset G\left(e_{K}\right)$ and $B e_{K}$ is a Galois extension with Galois group $G\left(e_{K}\right)$ where $N(K)$ is the normalizer of $K$ in $G$. A necessary and sufficient condition for $G\left(e_{K}\right)=N(K)$ is also given so that $B e_{K}$ is a Galois extension of $\left(B e_{K}\right)^{K}$ with Galois group $K$, and $\left(B e_{K}\right)^{K}$ is a Galois extension of $\left(B e_{K}\right)^{G\left(e_{K}\right)}$ with Galois group $G\left(e_{K}\right) / K$. Let $S(K)=\left\{H \mid H\right.$ is a subgroup of $G$ and $\left.e_{H}=e_{K}\right\}$. Then the map $S(K) \rightarrow e_{K}$ from $\{S(K) \mid K$ is a subgroup of $G\}$ to $B_{a}$ is one-to-one. In particular, when $K=G$, we derive an expression for $B, B=B e_{G} \oplus B\left(1-e_{G}\right)$ such that $B e_{G}=\oplus \sum_{i=1}^{m} B e_{i}$, a direct sum of central Galois algebras with Galois subgroup $H_{i}$, and $B\left(1-e_{G}\right)=B\left(1-\sum_{i=1}^{m} e_{i}\right)=C\left(1-e_{G}\right)$ which is a commutative Galois algebra with Galois group induced by and isomorphic with $G$. Moreover, a characterization for a Galois extension $B$ is shown in terms of the Galois extension $B e_{G}$ and $B\left(1-e_{G}\right)$. 
2. Definitions and notation. Let $B$ be a ring with $1, C$ the center of $B, G$ an automorphism group of $B$ of order $n$ for some integer $n$, and $B^{G}$ the set of elements in $B$ fixed under each element in $G$. We call $B$ a Galois extension of $B^{G}$ with Galois group $G$ if there exist elements $\left\{a_{i}, b_{i}\right.$ in $\left.B, i=1,2, \ldots, m\right\}$ for some integer $m$ such that $\sum_{i=1}^{m} a_{i} g\left(b_{i}\right)=\delta_{1, g}$ for each $g \in G$. We call $B$ a Galois algebra over $B^{G}$ if $B$ is a Galois extension of $B^{G}$ which is contained in $C$, and $B$ a central Galois extension if $B$ is a Galois extension of $C$. Throughout this paper, we will assume that $B$ is a Galois algebra with Galois group $G$. Let $J_{g}=\{b \in B \mid b x=g(x) b$ for all $x \in B\}$. In [2], it was shown that $B J_{g}=B e_{g}$ for some central idempotent $e_{g}$ of $B$. We denote by $\left(B_{a} ; \dot{+}, \cdot\right)$ the Boolean algebra generated by $\left\{0, e_{g} \mid g \in G\right\}$ where $e \cdot e^{\prime}=e e^{\prime}$ and $e \dot{+} e^{\prime}=e+e^{\prime}-e e^{\prime}$ for any $e$ and $e^{\prime}$ in $B_{a}$. Throughout, $e+e^{\prime}$ for $e, e^{\prime} \in B_{a}$ means the sum in the Boolean algebra $\left(B_{a} ; \dot{+}, \cdot\right)$ and a monomial $e$ in $B_{a}$ is $\Pi_{g \in S} e_{g} \neq 0$ for some $S \subset G$.

3. Galois extensions generated by idempotents. Let $K$ be a subgroup of $G$. The idempotent $\sum_{g \in K, e_{g} \neq 1} e_{g} \in B_{a}$ is called the group idempotent of $K$ denoted by $e_{K}$. Let $G(e)=\{g \in G \mid g(e)=e\}$ for $e \in B_{a}$. Then we will show that $K \subset G\left(e_{K}\right)$ and $e_{K}$ generates a Galois extension $B e_{K}$ with Galois group $G\left(e_{K}\right)$. A necessary and sufficient condition for $G\left(e_{K}\right)=N(K)$ is also given where $N(K)$ is the normalizer of $K$ in $G$. Thus some consequences for the Galois extension $B e_{K}$ can be derived when $K$ is a normal subgroup of $G$ or $K=G$.

LEMMA 3.1. For any $g, h \in G$,

(1) $g\left(e_{h}\right)=e_{g h g^{-1}}$.

(2) $e_{h}=1$ if and only if $e_{g h g^{-1}}=1$.

Proof. (1) It is easy to check that $g\left(J_{h}\right)=J_{g h g^{-1}}$, so $B g\left(e_{h}\right)=g\left(B e_{h}\right)=g\left(B J_{h}\right)=$ $B g\left(J_{h}\right)=B J_{g h g^{-1}}=B e_{g h g^{-1}}$. Thus $g\left(e_{h}\right)=e_{g h g^{-1}}$.

(2) It is clear by (1).

THEOREM 3.2. Let $K$ be a subgroup of $G, e_{K}=\sum_{g \in K, e_{g} \neq 1} e_{g}$, and $G\left(e_{K}\right)=\{g \in G \mid$ $\left.g\left(e_{K}\right)=e_{K}\right\}$. Then

(1) $K$ is a subgroup of $G\left(e_{K}\right)$ and

(2) $B=B e_{K} \oplus B\left(1-e_{K}\right)$ such that $B e_{K}$ and $B\left(1-e_{K}\right)$ are Galois extensions with Galois group induced by and isomorphic with $G\left(e_{K}\right)$.

Proof. (1) For any $g \in K$, by Lemma 3.1,

$$
\begin{aligned}
g\left(e_{K}\right) & =g\left(\sum_{\substack{k \in K \\
e_{k} \neq 1}} e_{k}\right)=\sum_{\substack{k \in K \\
e_{k} \neq 1}} g\left(e_{k}\right) \\
& =\sum_{\substack{k \in K \\
e_{k} \neq 1}} e_{g k g^{-1}}=\sum_{\substack{g k g^{-1} \in g K g^{-1} \\
e_{g} k g^{-1} \neq 1}} e_{g k g^{-1}}=e_{g K g^{-1}} .
\end{aligned}
$$

Since $g \in K, g K^{-1}=K$. Hence $g\left(e_{K}\right)=e_{K}$, and so $g \in G\left(e_{K}\right)$.

(2) We first claim that for any $e \neq 0$ in $B_{a}, B e$ is a Galois extension with Galois group induced by and isomorphic with $G(e)$. In fact, since $B$ is a Galois extension with Galois group $G$, there exists a $G$-Galois system for $B\left\{a_{i}, b_{i}\right.$ in $\left.B, i=1,2, \ldots, m\right\}$ for some 
integer $m$ such that $\sum_{i=1}^{m} a_{i} g\left(b_{i}\right)=\delta_{1, g}$ for each $g \in G$. Hence $\sum_{i=1}^{m}\left(a_{i} e\right) g\left(b_{i} e\right)=e \delta_{1, \mathfrak{g}}$ for each $g \in G(e)$. Therefore, $\left\{a_{i} e, b_{i} e\right.$ in $\left.B e, i=1,2, \ldots, m\right\}$ is a $G(e)$-Galois system for $B e$, and $e=\sum_{i=1}^{m}\left(a_{i} e\right)\left(b_{i} e-g\left(b_{i} e\right)\right)$ for each $g \neq 1$ in $G(e)$. But $e \neq 0$, so $\left.g\right|_{B e} \neq 1$ whenever $g \neq 1$ in $G(e)$. Thus, Be is a Galois extension with Galois group induced by and isomorphic with $G(e)$. Statement (2) is a particular case when $e=e_{K}$ and $e=1-e_{K}$, respectively.

The proof of Theorem 3.2(2) suggests an equivalence condition for a Galois extension $B$.

THEOREM 3.3. The extension $B$ is a Galois extension with Galois group $G(e)$ for a central idempotent e of $B$ if and only if $B=B e \oplus B(1-e)$ such that $B e$ and $B(1-e)$ are Galois extensions with Galois group induced by and isomorphic with $G(e)$. In particular, $B$ is a Galois algebra with Galois group $G(e)$ for a central idempotent e of $B$, if and only if $B=B e \oplus B(1-e)$ such that $B e$ and $B(1-e)$ are Galois algebras with Galois group induced by and isomorphic with $G(e)$.

Proof. $(\Rightarrow)$ Since $B$ is a Galois extension with Galois group $G(e), B=B e \oplus B(1-e)$ such that $B e$ and $B(1-e)$ are Galois extensions with Galois group induced by and isomorphic with $G(e)$ by the proof of Theorem 3.2(2).

$(\Leftarrow)$ Let $\left\{a_{j}^{(1)} ; b_{j}^{(1)} \in B e \mid j=1,2, \ldots, n_{1}\right\}$ be a $G(e)$-Galois system for $B e$ and let $\left\{a_{j}^{(2)} ; b_{j}^{(2)} \in B(1-e) \mid j=1,2, \ldots, n_{2}\right\}$ be a $G(e)$-Galois system for $B(1-e)$. Then we claim that $\left\{a_{j}^{(i)} ; b_{j}^{(i)} \mid j=1,2, \ldots, n_{i}, i=1,2\right\}$ is a $G(e)$-Galois system for $B$. In fact, $\sum_{i=1}^{2} \sum_{j=1}^{n_{i}} a_{j}^{(i)} b_{j}^{(i)}=e+(1-e)=1$. Moreover, for each $g \neq 1$ in $G(e)$-noting that $g \neq 1$ in $G(e)$ if and only if $\left.g\right|_{B e} \neq 1$ and $\left.g\right|_{B(1-e)} \neq 1$ by hypothesis-we have that $\sum_{j=1}^{n_{i}} a_{j}^{(i)} g\left(b_{j}^{(i)}\right)=0, i=1,2$, so $\sum_{i=1}^{2} \sum_{j=1}^{n_{i}} a_{j}^{(i)} g\left(b_{j}^{(i)}\right)=0$. Therefore $\left\{a_{j}^{(i)} ; b_{j}^{(i)} \mid j=\right.$ $\left.1,2, \ldots, n_{i}, i=1,2\right\}$ is a $G(e)$-Galois system for $B$, and so $B$ is a Galois extension with Galois group $G(e)$.

Next, it is clear that $B^{G(e)} \subset C$ if and only if $(B e)^{G(e)} \subset C e$ and $(B(1-e))^{G(e)} \subset$ $C(1-e)$, so by the above argument, $B$ is a Galois algebra with Galois group $G(e)$ for a central idempotent $e$ of $B$ if and only if $B=B e \oplus B(1-e)$ such that $B e$ and $B(1-e)$ are Galois algebras with Galois group induced by and isomorphic with $G(e)$.

COROLLARY 3.4. An algebra $B$ is a Galois algebra with Galois group $G$ if and only if $B=B e_{G} \oplus B\left(1-e_{G}\right)$ such that $B e_{G}$ and $B\left(1-e_{G}\right)$ are Galois algebras with Galois group induced by and isomorphic with $G$.

Proof. By Theorem 3.2(1), $G\left(e_{G}\right)=G$, so the corollary is immediate by Theorem 3.3.

Now let $S(K)=\left\{H \mid H\right.$ is a subgroup of $G$ and $\left.e_{H}=e_{K}\right\}$ and $\alpha: S(K) \rightarrow e_{K}$. It is easy to see that $\alpha$ is a bijection from $\{S(K) \mid K$ is a subgroup of $G\}$ to the set of group idempotents in $B_{a}$.

We are interested in an equivalence condition for $K$ such that $G\left(e_{K}\right)=N(K)$. We need the following lemma.

LEMмA 3.5. Let $K$ be a subgroup of $G$, then for a $g \in G, g \in G\left(e_{K}\right)$ if and only if $g K g^{-1} \in S(K)$. 
Proof. Suppose $g \in G\left(e_{K}\right)$, then

$$
\begin{aligned}
e_{K}=g\left(e_{K}\right) & =g\left(\sum_{\substack{k \in K \\
e_{k} \neq 1}} e_{k}\right)=\sum_{\substack{k \in K \\
e_{k} \neq 1}} g\left(e_{k}\right) \\
& =\sum_{\substack{k \in K \\
e_{k} \neq 1}} e_{g k g^{-1}}=\sum_{\substack{g k g^{-1} \in g K g^{-1} \\
e_{g} k g^{-1} \neq 1}} e_{g k g^{-1}}=e_{g K g^{-1}} .
\end{aligned}
$$

Thus $g K g^{-1} \in S(K)$. On the other hand, suppose $g K g^{-1} \in S(K)$. Then

$$
\begin{aligned}
g\left(e_{K}\right) & =g\left(\sum_{\substack{k \in K \\
e_{k} \neq 1}} e_{k}\right)=\sum_{\substack{k \in K \\
e_{k} \neq 1}} g\left(e_{k}\right) \\
& =\sum_{\substack{k \in K \\
e_{k} \neq 1}} e_{g k g^{-1}}=\sum_{\substack{g k g^{-1} \in g K g^{-1} \\
e_{g} k g^{-1} \neq 1}} e_{g k g^{-1}}=e_{g K g^{-1}}=e_{K} .
\end{aligned}
$$

Thus $g \in G\left(e_{K}\right)$.

THEOREM 3.6. $G\left(e_{K}\right)=N(K)$ if and only if $S(K)$ contains exactly one conjugate of the subgroup $K$.

Proof. $(\Rightarrow)$ For any $g \in G$ such that $g K g^{-1} \in S(K), g \in G\left(e_{K}\right)$ by Lemma 3.5. But $G\left(e_{K}\right)=N(K)$ by hypothesis, so $g \in N(K)$. Hence $g K g^{-1}=K$. Thus $S(K)$ contains exactly one conjugate of the subgroup $K$.

$(\Leftarrow)$ For any $g \in N(K), g K^{-1}=K$, so $g K g^{-1} \in S(K)$. Hence $g \in G\left(e_{K}\right)$ by Lemma 3.5. Thus $N(K) \subset G\left(e_{K}\right)$. Conversely, for each $g \in G\left(e_{K}\right), g K g^{-1} \in S(K)$ by Lemma 3.5, so $g K g^{-1}=K$ by hypothesis. Thus $g \in N(K)$. This implies that $G\left(e_{K}\right)=N(K)$.

COROLLARY 3.7. Assume that the order of $G$ is a unit in B. If $S(K)$ contains exactly one conjugate of the subgroup $K$, then $B e_{K}$ is a Galois extension of $\left(B e_{K}\right)^{K}$ with Galois group $K$ and $\left(B e_{K}\right)^{K}$ is a Galois extension of $\left(B e_{K}\right)^{G\left(e_{K}\right)}$ with Galois group $G\left(e_{K}\right) / K$.

Proof. By Theorem 3.2(2), $B e_{K}$ is a Galois extension with Galois group $G\left(e_{K}\right)$. Hence $B e_{K}$ is a Galois extension of $\left(B e_{K}\right)^{K}$ with Galois group $K$ for $K$ is a subgroup of $G\left(e_{K}\right)$ by Theorem 3.2(1). Moreover, by hypothesis, the order of $G$ is a unit in $B$, so the order of $K$ is a unit in $B e_{K}$. Since $S(K)$ contains exactly one conjugate of the subgroup $K, K$ is a normal subgroup of $G\left(e_{K}\right)$ by Theorem 3.6. Thus $\left(B e_{K}\right)^{K}$ is a Galois extension of $\left(B e_{K}\right)^{G\left(e_{K}\right)}$ with Galois group $G\left(e_{K}\right) / K$.

Next are some consequences for an abelian group $G$ or $K=G$.

COROLLARY 3.8. If $B$ is an abelian extension with Galois group $G$ (i.e., $G$ is abelian) of an order invertible in $B$, then for any subgroup $K$ of $G, B e_{K}$ is a Galois extension of $\left(B e_{K}\right)^{K}$ with Galois group $K$ and $\left(B e_{K}\right)^{K}$ is a Galois extension of $\left(B e_{K}\right)^{G\left(e_{K}\right)}$ with Galois group $G\left(e_{K}\right) / K$.

When $K=G$, we derive an expression for $B$ by using the set $\left\{e_{i} \mid i=1,2, \ldots, m\right\}$ of minimal idempotents in $B_{a}$. This gives detail descriptions of the components $B e_{G}$ and $B\left(1-e_{G}\right)$ as given in Corollary 3.4. 
TheOREM 3.9. Let $B$ be a Galois algebra with Galois group $G$. Then $B=B e_{G} \oplus$ $B\left(1-e_{G}\right)$ such that $B e_{G}=\oplus \sum_{i=1}^{m} B e_{i}$ where each $B e_{i}$ is a central Galois algebra with Galois group $H_{i}$ for some subgroup $H_{i}$ of $G$ and $B\left(1-e_{G}\right)=C\left(1-e_{G}\right)$ which is a commutative Galois algebra with Galois group induced by and isomorphic with $G$ in case $e_{G} \neq 1$ where $\left\{e_{i} \mid i=1,2, \ldots, m\right\}$ are given in $[5$, Theorem 3.8].

Proof. Since $e_{i}=\Pi_{h \in H_{i}} e_{h}$ where $H_{i}$ is the maximal subset (subgroup) of $G$ such that $\Pi_{h \in H_{i}} e_{h} \neq\{0\}$ or $e_{i}=\left(1-\sum_{j=1}^{t} e_{j}\right) \Pi_{h \in H_{i}} e_{h}$ where $H_{i}$ is the maximal subset (subgroup) of $G$ for some $t<i$ such that $\left(1-\sum_{j=1}^{t} e_{j}\right) \Pi_{h \in H_{i}} e_{h} \neq\{0\}$ (see [5, Theorem 3.8]), we have that $e_{i}\left(\sum_{g \in G, e_{g} \neq 1} e_{g}\right)=e_{i}$ for each $i$. Thus $\sum_{i=1}^{m} e_{i} \leq \sum_{g \in G, e_{g} \neq 1} e_{g}$. Noting that $e_{g}\left(1-\sum_{i=1}^{m} e_{i}\right)=0$ for each $g \neq 1$ in $G$ (see [5, Theorem 3.8]), we have that $\left(\sum_{g \in G, e_{\mathfrak{g}} \neq 1} e_{g}\right)\left(1-\sum_{i=1}^{m} e_{i}\right)=0$, that is, $\left(\sum_{g \in G, e_{\mathfrak{g}} \neq 1} e_{g}\right)\left(\sum_{i=1}^{m} e_{i}\right)=\sum_{g \in G, e_{\mathfrak{g}} \neq 1} e_{g}$. Hence $\sum_{g \in G, e_{g} \neq 1} e_{g} \leq \sum_{i=1}^{m} e_{i}$. Thus $\sum_{g \in G, e_{g} \neq 1} e_{g}=\sum_{i=1}^{m} e_{i}$, that is, $e_{G}=\sum_{i=1}^{m} e_{i}$. But then by [5, Theorem 3.8], $B=\oplus \sum_{i=1}^{m} B e_{i} \oplus B\left(1-\sum_{i=1}^{m} e_{i}\right)=B e_{G} \oplus B\left(1-e_{G}\right)$ such that $B\left(1-e_{G}\right)=C\left(1-e_{G}\right)$ which is a commutative Galois algebra with Galois group induced by and isomorphic with $G$, and $B e_{G}=\oplus \sum_{i=1}^{m} B e_{i}$ such that each $B e_{i}$ is a central Galois algebra with Galois group $H_{i}$ for some subgroup $H_{i}$ of $G$ where $\left\{e_{i} \mid i=1,2, \ldots, m\right\}$ are minimal idempotents of $B_{a}$.

4. A relationship between idempotents. In this section, we show a relationship between the set of idempotents $\left\{e_{g} \mid g \in G\right\}$ and the set of minimal elements in $B_{a}$, and give an equivalence condition for a monomial idempotent $e_{S}\left(=\sum_{g \in S, e_{g} \neq 1} e_{g}\right)$ where $S$ is a subset of $G$, and a monomial $e$ in $B_{a}$ is $\Pi_{g \in S} e_{g} \neq 0$ for some $S \subset G$.

THEOREM 4.1. Let $S$ be a subset of $G$. Then there exists a unique subset $Z_{S}$ of the set $\{1,2, \ldots, m\}$ such that $e_{S}=\sum_{i \in Z_{S}} e_{i}$.

Proof. Since $C=\oplus \sum_{i=1}^{m} C e_{i} \oplus C f$ (see [5, Theorem 3.8]), $e_{S}=\sum_{i=1}^{m} c_{i} e_{i}+c f$ for some $c_{i}, c \in C$. It can be checked that $e_{i}$ are minimal elements of $B_{a}$, so $e_{S} e_{i}=e_{i}$ or $e_{S} e_{i}=0$. Let $Z_{S}=\left\{i \mid e_{S} e_{i}=e_{i}\right\}$. Then for each $i \in Z_{S}, e_{i}=e_{S} e_{i}=c_{i} e_{i}$, and for each $i \notin Z_{S}, 0=e_{S} e_{i}=c_{i} e_{i}$. Hence $e_{S}=\sum_{i \in Z_{S}} e_{i}+c f$. Moreover, since $e_{g} f=0$ for each $g \neq 1$ in $G$ (see [5, Theorem 3.8]), we have that $0=e_{S} f=\left(\sum_{i \in Z_{S}} e_{i}+c f\right) f=c f$. Hence $e_{S}=\sum_{i \in Z_{S}} e_{i}$. The uniqueness of $Z_{S}$ is clear.

Next is a description of the components $B e_{K}$ and $B\left(1-e_{K}\right)$ for a subgroup $K$ of $G$ as given in Theorem 3.2.

COROLlary 4.2. For any subgroup $K$ of $G, B=B e_{K} \oplus B\left(1-e_{K}\right)$ such that $B e_{K}=$ $\sum_{i \in Z_{K}} B e_{i}$ and $B\left(1-e_{K}\right)=B\left(1-\sum_{i \in Z_{K}} e_{i}\right)$ which are Galois extensions with Galois group induced by and isomorphic with $G\left(e_{K}\right)$.

Proof. It is an immediate consequence of Theorems 3.2(2) and 4.1.

In [4], let $K$ be a subgroup of $G$. Then $K$ is called a nonzero subgroup of $G$ if $\prod_{k \in K} e_{k} \neq 0$, and $K$ is called a maximal nonzero subgroup of $G$ if $K \subset K^{\prime}$ where $K^{\prime}$ is a nonzero subgroup of $G$ such that $\prod_{k \in K} e_{k}=\prod_{k \in K^{\prime}} e_{k}$, then $K=K^{\prime}$. It was shown that the set of monomials in $B_{a}$ and the set of maximal nonzero subgroups of $G$ are in a one-to-one correspondence (see [4, Theorem 3.2]). Also, any maximal nonzero 
subgroup $K=H_{e}=\left\{g \in G \mid e \leq e_{g}\right\}$ where $e=\Pi_{k \in K} e_{k}$ and $H_{e}$ is a normal subgroup of $G(e)$ (see [4, Lemma 3.3]). Next is a characterization of a monomial idempotent $e_{S}$ $\left(=\sum_{g \in S, e_{g} \neq 1} e_{g}\right)$ for a subset of $G$.

THEOREM 4.3. Let $S$ be a subset of $G$ such that $e_{S}=\sum_{g \in S, e_{g} \neq 1} e_{g} \neq 0,1$. Then $e_{S}$ is a monomial if and only if $e_{j} \leq e_{S}$ whenever $H_{e_{S}} \subset H_{e_{j}}$ for an atom $e_{j}$.

Proof. $(\Rightarrow)$ By [4, Theorem 3.2], $e \rightarrow H_{e}$ is a one-to-one correspondence between the set of monomials in $B_{a}$ and the set of maximal nonzero subgroups of $G$. Noting that $e=\Pi_{g \in H_{e}} e_{g}$ when $e$ is a monomial, we have for any monomials $e$ and $e^{\prime}, H_{e} \subset H_{e^{\prime}}$ implies that $e \geq e^{\prime}$. Thus, $e_{j} \leq e_{S}$ whenever $H_{e_{S}} \subset H_{e_{j}}$ for an atom $e_{j}$ because $e_{S}$ is a monomial by hypothesis.

$(\Leftarrow)$ By Theorem 4.1, $e_{S}=\sum_{e_{i} \in Z_{S}} e_{i}$ where $Z_{S}=\left\{e_{i} \mid e_{i} \leq e_{S}\right\}$. Let $e=\Pi_{g \in H_{e_{S}}} e_{g}$. Then $e_{S} \leq e$ and $H_{e_{S}}=H_{e}$. Suppose $e_{S} \neq e$. Then $e_{S}=\sum_{e_{i} \in Z_{S}} e_{i}<e=\sum e_{j}$ where $\sum_{e_{i} \in Z_{S}} e_{i}$ is a direct summand of $\sum e_{j}$ by Theorem 4.1. It is easy to check that $H_{e_{S}}=\cap_{e_{i} \in Z_{S}} H_{e_{i}}=$ $H_{e}=\cap H_{e_{j}}$. Therefore there exists some $e_{j} \notin Z_{S}$, that is, $e_{j} \npreceq e_{S}$ such that $H_{e_{S}} \subset H_{e_{j}}$. This is a contradiction. Thus $e_{S}=e$, which is a monomial.

ACKNOWLEDGEMENTS. This paper was written under the support of a Caterpillar Fellowship at Bradley University. The authors would like to thank the Caterpillar Inc. for the support.

\section{REFERENCES}

[1] F. DeMeyer, Separable polynomials over a commutative ring, Rocky Mountain J. Math. 2 (1972), no. 2, 299-310.

[2] T. Kanzaki, On Galois algebra over a commutative ring, Osaka J. Math. 2 (1965), 309-317.

[3] G. Szeto, A characterization of Azumaya algebras, J. Pure Appl. Algebra 9 (1976), no. 1, 65-71.

[4] G. Szeto and L. Xue, The Boolean algebra and central Galois algebras, Int. J. Math. Math. Sci. 28 (2001), no. 4, 237-242.

[5] _ The structure of Galois algebras, J. Algebra 237 (2001), no. 1, 238-246.

[6] O. E. Villamayor and D. Zelinsky, Galois theory with infinitely many idempotents, Nagoya Math. J. 35 (1969), 83-98.

George Szeto: Department of Mathematics, Bradley University, Peoria, IL 61625, USA E-mail address: szeto@hi11top.brad1 ey.edu

Lianyong Xue: Department of Mathematics, Bradley University, Peoria, IL 61625, USA E-mail address: 1xue@hi 17top.brad1ey.edu 


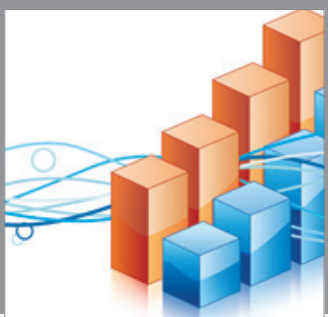

Advances in

Operations Research

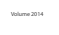

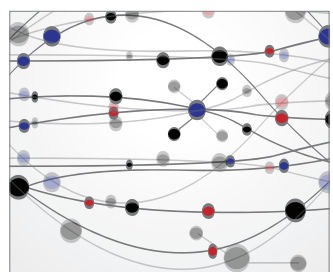

\section{The Scientific} World Journal
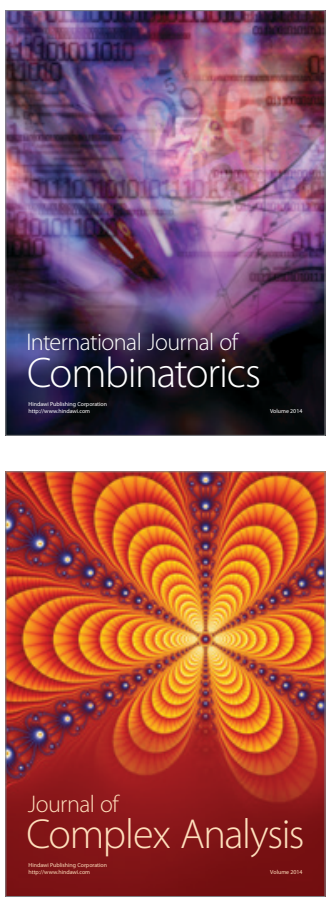

International Journal of

Mathematics and

Mathematical

Sciences
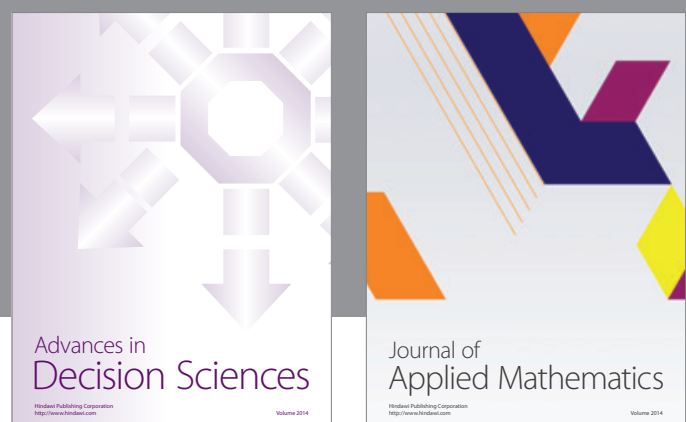

Journal of

Applied Mathematics
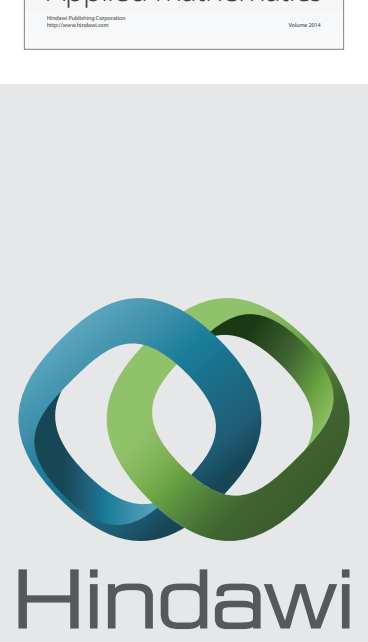

Submit your manuscripts at http://www.hindawi.com
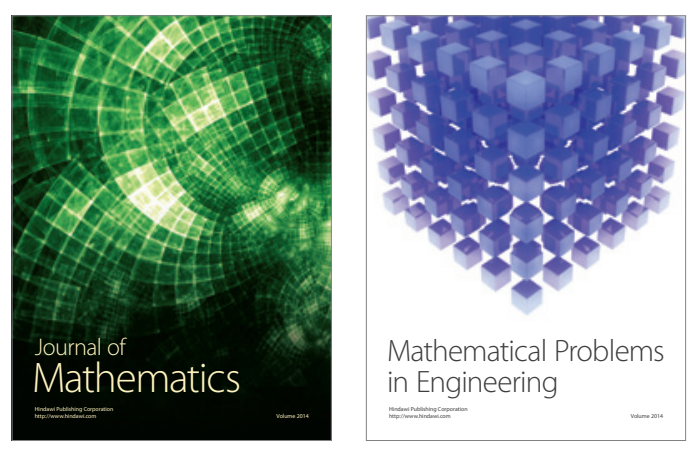

Mathematical Problems in Engineering
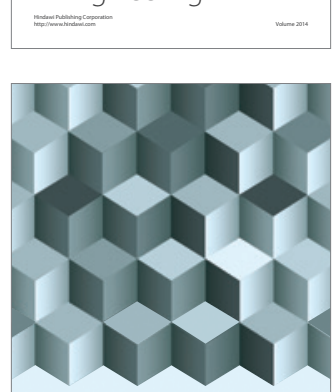

Journal of

Function Spaces
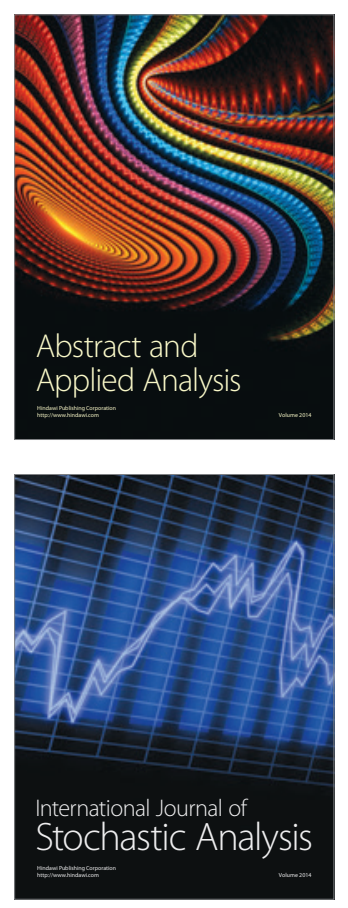

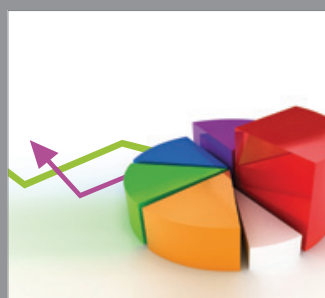

ournal of

Probability and Statistics

Promensencen
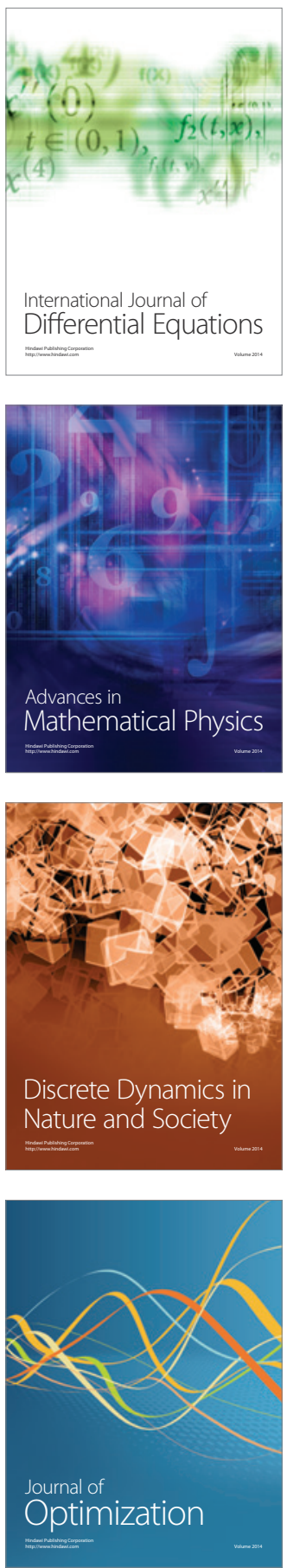